\title{
Prevalence and Associated Factors of Exclusive Breastfeeding Practices Among Mothers of Infants Less Than Six Months in Galkayo, Somalia: A Community Based Cross-Sectional Study
}

\author{
Adam Abdulkadir Mohamed ${ }^{1,2}$, Said Aden Mohamud ${ }^{1,2}$, Farhan Yahye Mohamud ${ }^{1,2,}$ \\ Khadra Abdirahman Ali ${ }^{2}$, Fadumo Ahmed Abdulle ${ }^{1}$, Aisha Abdikarim Hussein ${ }^{2}$ \\ ${ }^{1}$ Department of Public Health, Faculty of Health Sciences, Global Science University, Galkayo, Somalia \\ ${ }^{2}$ Department of Midwifery, Faculty of Health Sciences, Global Science University, Galkayo, Somalia
}

Email address:

adamafrican@gmail.com (A. A. Mohamed), sayidsaidgf@gmail.com (S. A. Mohamud), yahie03@gmail.com (F. Y. Mohamud), Khadra.jarato@gmail.com (K. A. Ali), faadumoahmed05@gmail.com (F. A. Abdulle),drscuud18@gmail.com (A. A. Hussein)

\section{To cite this article:}

Adam Abdulkadir Mohamed, Said Aden Mohamud, Farhan Yahye Mohamud, Khadra Abdirahman Ali, Fadumo Ahmed Abdulle, Aisha Abdikarim Hussein. Prevalence and Associated Factors of Exclusive Breastfeeding Practices Among Mothers of Infants Less Than Six Months in Galkayo, Somalia: A Community Based Cross-Sectional Study. Science Journal of Public Health.

Vol. 6, No. 5, 2018, pp. 133-139. doi: 10.11648/j.sjph.20180605.13

Received: October 7, 2018; Accepted: October 19, 2018; Published: November 13, 2018

\begin{abstract}
Background: Exclusive breastfeeding has crucial importance on the lives of infants and young children. It provides all the energy and nutrients that infant needs for the first months of life. In sub-Saharan African countries including Somalia, the morbidity and mortality rates of infants and young children less than five years of age are high compared to developed countries. The main causes of these are mainly due to inadequate and sufficient EBF/BF practices with the combination of diseases like infant diarrhea, pneumonia, malaria and measles. Thus this study was aimed to assess the prevalence and associated factors of EBF in Somalia. Methods: A community based cross-sectional study was conducted. Data were collected from 384 mothers of infants less than 6 months of age residing in Northern side of Galkayo District using systematic sampling method. Cumulative Odds Ratio with $95 \%$ confidence interval was estimated using bivariate analysis with binary logistic regression model to identify independent determinants of exclusive breastfeeding practice. Results: Majority (99.7\%) of the interviewed mothers had ever breastfed their baby. The prevalence of exclusive breastfeeding was $5.2 \%$ whereas $94.8 \%$ did not exclusively breastfeed their baby up to the intended six months. A mother with formal education was a predictor to exclusive breastfeeding practice. Having formal education, health facility delivery, receiving EBF counseling and maternal unemployment were found to be predictors of exclusive breastfeeding practice. Conclusion: The prevalence of exclusive breastfeeding was found to be very low compared to the WHO recommendations $(5.2 \%$ in our study compared to $90 \%$ of WHO recommendations). However, our current study found that, majority ( $\mathrm{n}=221,57.5 \%)$ of the women had knowledge on exclusive breastfeeding initiation. We recommend to strengthen the available basic services and to promote formal education on mothers in Somalia. We also recommend means to encourage mothers to deliver health facilities and to increase the awareness and counseling of EBF among pregnant mothers during their visit of ANC.
\end{abstract}

Keywords: Exclusive Breastfeeding, Determinants, Somalia

\section{Introduction}

Exclusive breastfeeding has been defined by WHO as "the situation where the infant has received only breast milk from his/her mother or a wet nurse or expressed milk until six months and no other liquids/solids with the exception of drops or syrups consisting of vitamins ,mineral supplements or medicine" [1]. EBF for the first six months of infants' life is a cost effective intervention in saving children's lives and it is recommended by the world Health Organization and UNICEF [1, 14]. With exclusive breastfeeding universal coverage of $90 \%$, approximately the deaths $13 \%$ to $14 \%$ of infants and young children less than five years of age can be prevented in low and middle income countries [2]. Babies 
who have been breastfed exclusively have shown to have lower rates of acute respiratory infections and diarrhea and to have a better neurodevelopment outcome and have better physical growth compared to mixed fed or non-breastfed infants [3].

Exclusive breastfeeding has crucial importance on the lives of infants and young children. It provides all the energy and nutrients that infant needs for the first months of life. Breastfeeding promotes growth and cognitive development of the child. Breast milk contains living cells and defensive factors which empowers the immune system therefore protecting the infant from infections and respiratory conditions. In many countries in the world including Somalia, the morbidity and mortality rates of infant and young children under five years of age are high. The main causes of these deaths are mainly due to inadequate breastfeeding practices with combination of diseases like infant diarrhea, pneumonia, malaria and measles [4].

Early initiation of breastmilk after delivery is highly recommended especially within the first hour after birth as it reduces neonatal mortality and morbidity. Colostrum, the "pre-milk" is a chock-full of antibodies to protect the newborn, its higher in protein and low in sugar to meet the needs of the newborn but after few days (usually three to four days) the breast milk becomes more in sugar and in volume than Colostrum because more calories and frequent feeding are needed to accelerate growth. Exclusive breastfeeding is important for the mothers too as it lowers their risks in postmenopausal osteoporosis and chances developing breast and ovarian cancers, breastfeeding the baby exclusively keeps the menstrual cycle at bay therefore causing lactation amenorrhea which could be a natural way of birth control [5]. Since in exclusive breastfeeding the infants are breastfed frequently, it increases mother-infant bonding. It is actually time for nursing, comfort and nurturing, a time for studying and memorizing each other's faces. Infants usually seek nursing in order to ease a pain, relieve stress or to regain security.

For all these reasons and many more, breastfeeding is a public health priority promoted and supported by 'The global strategy on infant young child feeding" approved by $55^{\text {th }}$ world health assembly in 2003. Factors that globally influence exclusive breastfeeding include maternal education, age of the mother, mothers' attendance at antenatal services during pregnancy, parity of the mother, mothers working outside home, mother's domestic work, mother's knowledge on infant and young child feeding and so on. Globally, only about $40 \%$ of infants aged $0-6$ months are exclusively breastfed [6]. Analyzes show that inappropriate breastfeeding practices and non-exclusive breastfeeding practices contribute to $11.6 \%$ which is equivalent to 804000 of mortality in children less than five years of age in 2011 [7]. Despite the World Health Assembly's (WHA) targets to increase exclusive breastfeeding by $50 \%$ in 2025 [6]. Somalia has some of the worst maternal health indicators in the world as malnutrition is chronic, early marriage is common, and most births are delivered at home without the presence of a skilled birth attendant (attended by TBAs). Women in Somalia live in a highly insecure context where healthcare infrastructure and maternal health programs have been disrupted and limited in availability for decades [8].

This study was aimed to generate information on prevalence and determining factors on exclusive breast feeding practices among mothers of children less than six months in Galkayo. The findings of this study w0uld be useful to the Ministry of Health (MOH), the NonGovernmental Organizations (NGOs) working in child survival programs in planning, implementing and in designing interventions to improve the practice of exclusive breastfeeding in the area and other similar circumstances. The findings would also be useful in the contribution to the ongoing research efforts o EBF and child survival. The results of this research would also promote further studies in the region and in other parts of the country and provide clinical data on exclusive breastfeeding. It would also Help Support effective strategies for improving public awareness regarding the benefits of EBF and to offer specific measures to government on how to enhance and sustain the EBF practice so as to promote child health and reduce child morbidity and mortality. The results of this study would lead to development of positive attitude and help to reduce public misperceptions on women's choices on EBF and provide more scientific evidence on importance of EBF to health care givers in advocating the practice.

\section{Materials and Methods}

\subsection{Study Area}

This study was conducted in northern side (Puntland) of Galkayo. Galkayo is the capital city of north-central Mudug region of Somalia. It is administratively divided into two areas separated by a distinct boundary with northern districts governed by the Puntland state and southern districts by Galmudug state. Galkayo is geographically divided into four main neighborhoods of Garsoor, Horumar, and Isra in the Puntland region and Wadajir in the Galmudug region. The district has considerably grown in the recent years since it is in a strategic location serving as a commercial hub with a population of about 545,000 . This study was conducted from March to august 2018.

\subsection{Study Participants and Sampling Method}

This study adopted a descriptive cross sectional, quantitative study to investigate prevalence exclusive breastfeeding and associated factors among infants less than 6 months in Galkayo Somalia. We used quantitative method because we wanted to collect all data about prevalence exclusive breastfeeding and associated factors among infants of 0-6 months in Galkayo Somalia in specific time in community based. Probability sampling method (systematic sampling) was used this study. Houses of Mothers with infants less than six months were first estimated $(10,000$. Estimation from the local government) and sample size of 
roughly 400. The sample fraction here was 400/10000= $1 / 25$, thus $\mathrm{k}=25$. The first house in the villages was selected randomly between 1 and 25 houses. The first and every 25 th house was subsequently selected as sample members until we reached out our intended sample size. All the mothers with children aged from 0-6 months living in the sampled villages were added in the study. Statistical packages for social science (SPSS) and STATA were used in data entry and analysis respectively.

\subsection{Sample Size and Data Collection Technique}

The study sample was taken on basis of the following formula for respondents. The sample size will be computed using Kish and Leslie formula (1996) which demonstrated as follow;

$\mathrm{n}=\frac{\mathrm{z}^{2} \mathrm{pq}}{\mathrm{d}^{2}}$ Where; $\mathrm{n}=$ Sample size, $\mathrm{z}=$ Confidence interval at $95 \%$ which is $1.96, \mathrm{p}=$ Proportion of the target population, $\mathrm{q}=$ the difference between 1 and $\mathrm{p}, \mathrm{d}=$ desired degree of accuracy considered. In order to conduct the study, the following assumptions were made; The proportion of women who exclusively breastfeed were taken to be $39 \%$ (EBF prevalence in Puntland) According UNICEF 2016 report [14]. 95\% confidence level (standard value 1.96), and Maximum margin of error of $5 \%$. Substituting into the formula, the sample size will be 384 mothers.

The data was collected using self-administered questionnaires the mothers. For those mothers who are able to read and write, the questionnaire was given to them and they filled it up by themselves freely, but for those who could not read and write or faced difficulties in understanding the question, they were assisted by the data collector.

\section{Results}

\subsection{Socio Demographic Determinants of Households}

A total of 384 mothers participated in the study, majority of the respondents $(n=188)$ were between $25-34$ years of age (49\%) and only nine mothers were 45 or above years of age during the interview. Majority of the infants $(n=225)$ were ages between 2 to 4 months of age $(58.6 \%)$. Although majority of the respondents were married (46.6\%), still significance portion of the participants were either divorced $(29.7 \%)$ or widowed $(23.7 \%)$. Majority of the mothers that participated this study, two hundred twelve $(55.2 \%)$ have no formal education compared to the one hundred seventy-two (44.8\%) who had formal education. Majority of the mothers/caregivers $(n=298)$ were unemployed and only $22.4 \%$ were employed. More than half of the families 195 $(50.8 \%)$ were living low income (less than 200 USD per month) and $162(42.2 \%)$ were living income between 200 to 400 USD per month. This implies that, the majority of the families were low in wealth.

Table 1. Socio-demographic characteristics of mothers with infants aged 0-6 months $(n=384)$, Galkayo District, Puntland, Somalia, June 2018.

\begin{tabular}{|c|c|c|c|}
\hline \multirow[t]{2}{*}{ Variable } & \multicolumn{3}{|l|}{ Characteristics } \\
\hline & Category & Frequency & Percentage \% \\
\hline \multirow{4}{*}{ Mother's Age (in years) } & $15-24$ & 136 & 35.4 \\
\hline & $25-34$ & 188 & 49.0 \\
\hline & $35-44$ & 51 & 13.3 \\
\hline & 45 and above & 9 & 2.3 \\
\hline \multirow{6}{*}{ Child Age (Months) } & Up to 1 month & 51 & 13.3 \\
\hline & 2 months & 73 & 19.0 \\
\hline & 3 months & 65 & 16.9 \\
\hline & 4 months & 87 & 22.7 \\
\hline & 5 months & 69 & 18.0 \\
\hline & 6 months & 39 & 10.1 \\
\hline \multirow{3}{*}{ Marital Status } & Divorced & 114 & 29.7 \\
\hline & Married & 179 & 46.6 \\
\hline & Widowed & 91 & 23.7 \\
\hline \multirow{2}{*}{ Education } & Formal Education & 172 & 44.8 \\
\hline & No formal education & 212 & 55.2 \\
\hline \multirow{2}{*}{ Maternal Employment } & Unemployed & 298 & 77.6 \\
\hline & Employed & 86 & 22.4 \\
\hline \multirow{2}{*}{ Place of last delivery } & Home & 180 & 46.9 \\
\hline & Health facility & 204 & 53.1 \\
\hline \multirow{2}{*}{ Mode of delivery } & Normal & 364 & 94.8 \\
\hline & C-Section & 20 & 5.2 \\
\hline \multirow{4}{*}{ Monthly Income (USD) } & Less than 200 & 195 & 50.8 \\
\hline & $201-400$ & 162 & 42.2 \\
\hline & $401-600$ & 22 & 5.7 \\
\hline & Above 600 & 5 & 1.3 \\
\hline
\end{tabular}

Source: Field Data 2018

\subsection{Mother's Place of Last Delivery}

Majority of the participants $(\mathrm{n}=204)$ delivered their last baby in health facility $(53.1 \%)$ while the rest $(\mathrm{n}=180)$ delivered their 
last baby in their homes attended by traditional birth attendants $(46.9 \%)$.

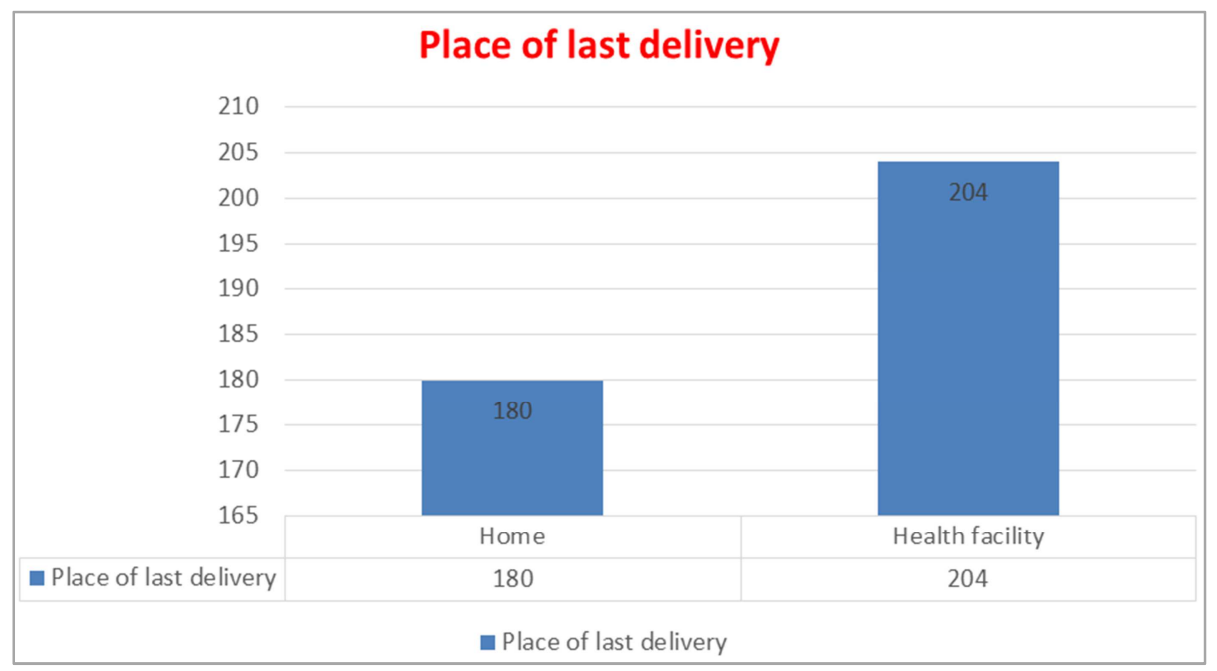

Figure 1. Place of last delivery of the respondents.

\subsection{Mode of Delivery}

On the issue of mode of the delivery, three hundred sixtyfour mothers $(95 \%)$ have spontaneous/normal delivery and the rest $5 \%$ delivered through $\mathrm{C}$-section.

\section{Mode of delivery}

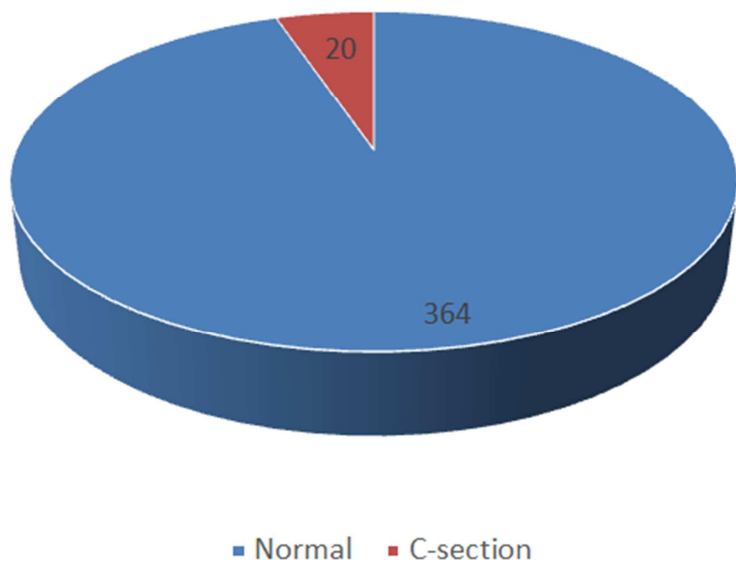

Figure 2. Mode of last delivery.

\subsection{Breastfeeding Indicators, Prevalence and Knowledge of the Respondents}

Majority of the interviewed mothers with infants less than six months $(n=383,99.7 \%)$ ever breastfeed their children and only one mother (0.03) did not ever breastfeed her child. The number of mothers who are exclusively breastfeeding $(n=20$, $5.2 \%$ ) were very low compared to those who are not exclusively breastfeeding their baby $(n=364,94.8 \%)$. Two hundred twenty-one $(57.5 \%)$ of the mothers/caregivers answered the right time to give breast milk after the infant was immediately delivered. Majority of the participant $(n=221)$ had poor knowledge and also majority of the mothers were with positive attitude towards EBF. One hundred ninety-eight (51.6\%) expressed that the first thing to do immediately after delivery is to feed with colostrum milk. One hundred seventy-nine $(46.6 \%)$ of the respondents strongly agreed that discarding the colostrum was important during delivery.

Nearly half of the mothers/caregivers $(n=168)$ answered the right time to start complementary foods were at 3 months of less than. This is also indicating that the majority of the respondents had low knowledge on the length of exclusive breastfeeding and the time to initiate complementary foods.

Table 2. Breastfeeding indicators, prevalence and Knowledge of the respondents.

\begin{tabular}{llll}
\hline \multirow{2}{*}{ Variable } & Characteristics & & \\
\cline { 2 - 4 } & Category & N & \% \\
\hline Exclusive breastfeeding & No & 364 & 94.8 \\
(<6 Months) & Yes & 20 & 5.2 \\
Child ever breastfeed & Yes & 383 & 99.7 \\
& No & 1 & 0.3 \\
Right time to give breast & Immediately & 221 & 57.5 \\
milk after the child is & Within an hour & 99 & 25.8 \\
delivered & Between 1 hour and 3 hours & 59 & 15.4 \\
& From 3 to 7 hours & 5 & 1.3 \\
Right thing to do with the & Discard & 179 & 46.6 \\
colostrum & Feed immediately & 198 & 51.6 \\
& I don't know & 7 & 1.8 \\
Right time to start & 3 months or less & 168 & 43.8 \\
complementary foods & 4 months & 64 & 16.7 \\
& 5 months & 40 & 10.4 \\
& 6 months & 67 & 17.4 \\
\hline
\end{tabular}

Source: Field Data 2018

Three hundred thirty-four (87\%) of the interviewed mothers did not get counseling towards whether EBF or BF in general. Only $13 \%$ received counseling of EBF during antenatal care or Post-natal care visits. The mothers declared that the main source of this counseling was from the health care workers. All mothers had ever breastfed their children at 
some point and they were breastfeeding their index infant during the time of the data collection. Majority of the respondents $(n=239,62.2 \%)$ answered that their baby received breast milk for the first time and one hundred fortyfive $(n=145,37.8 \%)$ of the mothers initiated plain and pure water to their baby after delivery. This is a good indication of that Galkayo mothers have low knowledge, awareness and counseling.

\subsection{Bivariate Analysis}

To determine which factors has association with maternal exclusive breastfeeding and which factors are not, we used bivariate analysis. Maternal educational status, maternal employment, place of delivery and prior EBF counseling were found to be determinant factors with the practice of exclusive breastfeeding. Infants less than 6 months with mother/caregivers who had formal education were 3.2 times more likely to be exclusively breastfed COR: $3.2,95 \%$ CI [ 1.6 , $4.1)]$ compared to infants whose mothers/caregivers had nonformal education. Children's of mothers who are employed were less likely to be exclusively breastfed COR: 95\%CI [ 0.5 , 2.2)] compared to children's of mothers/caregivers who are not employed. Mothers who delivered at health facilities were 5 times more likely to be exclusively breastfed COR: 5.1, $95 \%$ CI $(2.2,8.0)]$ compared to mothers who delivered at home/TBAs home. Mothers who received prior counseling towards exclusive breastfeeding practice were two times more likely to exclusively breastfeed COR: 95\% CI [(1.4, 4.2)] compared to mothers who do not get prior exclusive breastfeeding counseling during pregnancy or delivery. All the other socio-demographic factors did not show association with exclusive breastfeeding practice.

Table 3. Determinants of Exclusive Breastfeeding, Galkayo, Somalia 2018: Bivariate analysis.

\begin{tabular}{|c|c|c|c|c|c|c|c|}
\hline \multirow{2}{*}{ Variables } & & \multicolumn{4}{|l|}{ EBF } & \multirow{4}{*}{ P-value } & \multirow{4}{*}{ COR $(95 \% C I)$} \\
\hline & & \multirow{2}{*}{$\begin{array}{ll}\text { No } \\
\mathbf{N} \\
\end{array}$} & \multicolumn{3}{|c|}{ Yes } & & \\
\hline \multirow{2}{*}{ Total } & & & $\%$ & $\mathbf{N}$ & $\%$ & & \\
\hline & & 364 & 94.8 & 20 & 5.2 & & \\
\hline \multirow{2}{*}{ Educational status } & Formal & 156 & 90.7 & 16 & 9.3 & \multirow[t]{2}{*}{0.00} & $3.2(1.6,4.1)$ \\
\hline & Non-Formal & 208 & 98.1 & 4 & 1.9 & & 1 \\
\hline \multirow{2}{*}{ Employment } & Employed & 84 & 97.7 & 2 & 2.3 & \multirow[t]{2}{*}{0.105} & $0.7(0.5,2.2)$ \\
\hline & Unemployed & 280 & 94 & 18 & 6.0 & & 1 \\
\hline Place of delivery & Home & 175 & 97.2 & 5 & 2.8 & 0.00 & 1 \\
\hline \multirow{2}{*}{$\begin{array}{l}\text { Breastfeeding counseling } \\
\text { received }\end{array}$} & No & 322 & 96.4 & 12 & 3.6 & \multirow[t]{2}{*}{0.001} & $2.8(1.4,4.2)$ \\
\hline & Yes & 42 & 84 & 8 & 16.0 & & 1 \\
\hline
\end{tabular}

\section{Discussion, Conclusion and Recommendation}

\subsection{Discussion}

This study attempted to determine the prevalence and associated factors of exclusive breastfeeding practice among mothers with infants less than six months of age in Galkayo district. The prevalence of ever breastfeeding was $99.7 \%$ while the crude prevalence of exclusive breastfeeding was $94.8 \%$ and only $20(5.2 \%)$ mothers exclusively breastfed their infants. In the bivariate analysis, having formal education, being unemployed, delivering at health facility and receiving prior EBF counseling were found to be independent predictors of exclusive breastfeeding among the infants aged less than six months. In this study, maternal age, child age, marital status, knowledge, mode of delivery and parity were not a predictor to EBF.

In this study, Infants whose mothers had formal education were more likely to be exclusively breastfed. This was in line with study in Malawi which indicated that mothers with secondary education are four times more likely to exclusively breastfed compared to those with no or primary education, so formal education was positive determinant of exclusive breastfeeding [9]. Maternal educational status and exclusive breastfeeding showed significance association. this was in line with a study in Nigeria [10] which concluded that Maternal education below secondary level contributed to sub-optimal feeding and failure to practice exclusive breastfeeding.

This study has indicated a significant difference between employed and unemployed mothers with regard to exclusive breastfeeding in which employed mothers were less likely to exclusively breastfeed. This could be attributed to the fact of less maternity leave for the mother which gives the mother less opportunity to stay at home hence hindering exclusive breastfeeding. This could also be explained in the sense that most women in Galkayo are the bread winners for the family leaving their infants at home to be taken care by someone else usually by another child.

The mode of delivery did not show significant with exclusive breastfeeding in this study. Normal delivery (94.8\%) and caesarian section (5.2\%), This result was in line with a studies in Nigeria which conclude that the Caesarean delivery was usually associated with feeding the newborn with formula and low breast milk production $[11,15]$. It was also in alternate to a study of the effects of maternal care practice on breastfeeding by [12] which revealed that type of delivery (vaginal or caesarean) had no influence on breastfeeding practices. Women who delivered at a health facility practiced exclusive breastfeeding more compared to those who delivered at home. This could be explained in the sense that mothers received breastfeeding counseling from 
the health professionals after delivery.

Infants whose families had a low income (less than 200 dollars per month) or even with high income wealth did not show relationship/association with exclusive breastfeeding practice. This was constant with two studies from Ethiopia [13, 16]. When assessed the knowledge, attitude and early breastfeeding practices of the respondents, the study showed that $57.5 \%$ of the mother's breastfed their immediately after birth and $51 \%$ fed the baby with colostrum. This study showed that $43.8 \%$ mothers said that the right time to start complementary feeding was 3 months of age or less. This indicated that the majority of the respondents had low knowledge on the timeline of exclusive breastfeeding and the time to initiate complementary foods.

According to this study, a great significant number of the mothers $(87 \%)$ had not received any information/counseling regarding breastfeeding and young child feeding. and the only $13 \%$ mothers who received, received it from the health workers at health facility. This might be attributed to the fact that there was very low awareness in the community regarding exclusive breastfeeding and optimal breastfeeding practices, although the infant mortality rate was high in Galkayo.

\subsection{Strength and Limitation}

This study can be interpreted in light of its strengths and limitations. The use of validated questionnaires, the fact that this study assessed individual factors, including knowledge and attitude of mothers, as well as variables related to families can be considered as a major strength of this study. However, other factors like child birth weight, health status of the mother and the child and other unmentioned factors which might be associated with exclusive breast feeding were not addressed in this study. In addition, not using enough literatures from the surrounding towns on the study area in specific due to the unavailability of published journals on the topic of interest could be mentioned as a limitation. Furthermore, this study used a cross-sectional study design, which made it difficult to establish causal effect relationship.

\section{Conclusion}

Based on the findings, we conclude: That the prevalence of exclusive breastfeeding in our study for the age group 0-6 months in Galkayo were $5.2 \% \%(n=20)$ which is below the World Health Organization infant and young child feeding recommendations. Mothers who were unemployed, with formal education, those who received EBF counseling and those who delivered at health facility had considerably higher exclusive breastfeeding practice compared to those who were, employed, illiterate, delivered at homes and those who do not receive EBF counseling. Great number of the respondents did not receive breastfeeding information and counseling. Most of the mothers initiated breastfeeding immediately after delivery, fed the newborn with Colostrum immediately but started breastfeeding sup-optimal practices at 3 months of age or less. The hypothesis of this study was to test if there is association between the socio-demographic factors and exclusive breastfeeding. This study found that being married, having formal education, being unemployed, being delivered at health facility and receiving EBF counseling were all significant factors to EBF, thus the null hypothesis is rejected.

\section{Recommendations}

Government and interested bodies should develop strategies to improve awareness of EBF among women both at health facility and community level in order to improve EBF practice.

Health institutions should raise awareness and encourage pregnant women to attend health facility during delivery and increase breastfeeding counseling after delivery.

More information should be disseminated to mothers on infant and young child feeding timeline in regards when to start complementary feeding.

Policies on maternity leaves for working mothers should be revised.

\section{Disclosure}

All the authors do not have any possible conflicts of interest.

\section{References}

[1] WHO. 10 facts on child health. Geneva, 2012 https://www.who.int/features/factfiles/nutrition/en/.

[2] Jones, G., Steketee, R. W., Black, R. E., Bhutta, Z. A., \& Morris, S. S. (2003). How many child deaths can we prevent this year? Lancet. https://doi.org/10.1016/S01406736(03)13811-1.

[3] McFadden, A., \& Toole, G. (2006). Exploring women's views of breastfeeding: A focus group study within an area with high levels of socio-economic deprivation. Maternal and Child Nutrition, 2(3), 156-168. https://doi.org/10.1111/j.17408709.2006.00054.x.

[4] Ahmed, A. H. (2008). Breastfeeding preterm infants: an educational program to support mothers of preterm infants in Cairo, Egypt. Pediatric Nursing, 34(2), 125-130, 138. Retrieved http://www.ncbi.nlm.nih.gov/pubmed/18543837.

[5] Dieterich, C. M., Felice, J. P., O'Sullivan, E., \& Rasmussen, K. M. (2013). Breastfeeding and Health Outcomes for the Mother-Infant Dyad. Pediatric Clinics of North America. https://doi.org/10.1016/j.pcl.2012.09.010.

[6] World Health Organization, http://www.who.int/newsroom/fact-sheets/detail/infant-and-young-child-feeding.

[7] Black, R. E., Allen, L. H., Bhutta, Z. A., Caulfield, L. E., de Onis, M., Ezzati, M., ... Child Undernutr, S. (2008). Maternal and child undernutrition: global and regional exposures and health consequences. Lancet, 371(9608), 243-260. https://doi.org/10.1016/s0140-6736 (07) 61690-0. 
[8] Adam A. Mohamed, Abiodun O. Ilesanmi and M. David Dairo, The Experience of Women with Obstetric Fistula following Corrective Surgery: A Qualitative Study in Benadir and Mudug Regions, Somalia. Obstetrics and Gynecology International, Volume 2018, Article ID 5250843, 10 pages https://doi.org/10.1155/2018/5250843.

[9] Kamudoni, P., Maleta, K., Shi, Z., \& Holmboe-Ottesen, G. (2007). Infant feeding practices in the first 6 months and associated factors in a rural and semiurban community in Mangochi District, Malawi. Journal of Human Lactation, 23(4), 325-332. https://doi.org/10.1177/0890334407307567.

[10] Ajibade B L, Okunlade J O, Makinde o y, Amoo p o, A. m o. (2013). Factors Influencing the Practice of Exclusive Breast Feeding in Rural Communities of Osun State, Nigeria . European Journal of Business and Management, 5(15), 4954.

[11] Lawoyin, T. O., Olawuyi, J. F., \& Onadeko, M. O. (2001). Factors associated with exclusive breastfeeding in Ibadan, Nigeria. Journal of Human Lactation: Official Journal of International Lactation Consultant Association, 17(4), 321325 .

[12] DiGirolamo, A. M., Grummer-Strawn, L. M., \& Fein, S. B. (2008). Effect of Maternity-Care Practices on Breastfeeding. $\begin{array}{lll}\text { Pediatrics, } & \text { 122(Supplement } & \text { 2), }\end{array}$ https://doi.org/10.1542/peds.2008-1315e.

[13] Central Statistical Agency [Ethiopia] and ICF International. (2016). FEDERAL DEMOCRATIC REPUBLIC OF ETHIOPIA ETHIOPIA Demographic and Health Survey 2016 Key Indicators Report. Ethiopians Water Sector Development Program. Retrieved from https://www.usaid.gov/sites/default/files/documents/1860/Ethi opia DHS 2016 KIR - Final 10-17-2016.pdf.

[14] UNICEF 2016 Somali infant and Young child nutrition Assessment https://reliefweb.int/sites/reliefweb.int/files/resources/2016Somali-IYCN-Assessment-Final-Report.pdf.

[15] Adelekan, D. A. (2003). Childhood nutrition and malnutrition in Nigeria. Nutrition, 19(2), 179-181. https://doi.org/10.1016/S0899-9007(02)01101-2.

[16] Adugna, B., Tadele, H., Reta, F., \& Berhan, Y. (2017). Determinants of exclusive breastfeeding in infaless than six months of age in, 4-11. https://doi.org/10.1186/s13006-0170137-6. 